\title{
THE NOTION OF RESTRICTED IDĖLES WITH APPLICATION TO SOME EXTENSION FIELDS
}

\author{
YOSHIOMI FURUTA
}

Dedicated to the memory of Professor Tadasi Nakayama

Let $k$ be an algebraic number field of finite degree, $K$ be its normal extension of degree $n$, and $\hat{s}$ be the set of those primes of $K$ which have degree 1 . Using this set $\hat{s}$ instead of the set of all primes of $K$, we define an $\hat{s}$-restricted idèle of $K$ by the same way as ordinary idèles. It is known by Bauer that the normal extension of an algebraic number field is determined by the set of all primes of the ground field which are decomposed completely in the extension field. This suggests that if we treat abelian extensions over $K$ which are normal over $k$, the class field theory is expressed by means of the $\hat{s}$-restricted idèles (theorem 2). When $K=k, \hat{s}$ is the set of all primes of $K$, and we have the ordinary class field theory.

In fact, the $\mathfrak{P}$-completion $K_{\mathfrak{P}}$ is isomorphic to the $\mathfrak{p}$-completion $k_{\mathfrak{p}}$, when $\mathfrak{P}$ belongs to $\hat{s}$ and divides a prime $\mathfrak{p}$ of $k$. Therefore the group of all $\hat{s}$-restsicted idèles of $K$ is isomorphic to the direct product of $n$-fold of a group of restricted idèles of $k$. This means that the abelian extensions over $K$ which are normal over $k$ are determined by some objects ( $\hat{s}$-admissible subgroups of the $\hat{s}$-restricted idèle group) in the ground field $k$. In this paper we shall characterize this object by the connected component of the unity in the idèle class group of $K$ (theorem 3 ) and by the principal idèle group of $K$ (theorem 4). This result is a generalization of our preceeding paper $[8](\$ 8)$.

Throughout this paper the following notations will be used.

$F \quad$ an algebraic number field of finite degree.

$F^{\times} \quad$ the multiplicative group of all non zero elements of $F$, which is identified with the principal idèle group of $F$.

$F_{\mathfrak{p}}$ the $\mathfrak{p}$-adic completion of $F$, where $\mathfrak{p}$ is a finite or infinite prime of $F$.

$U_{\mathfrak{p}} \quad$ the p-adic unit group of $F$.

Received April 10, 1965. 


\author{
$J=J_{F} \quad$ the idèle group of $F$. \\ $J_{\infty} \quad$ the subgroup of all elements of $J$ which have components 1 at \\ all finite primes. \\ $\mathfrak{S}=\mathfrak{S}_{F}=J / F^{\times}$the idèle class group of $F$. \\ D the connected component of the unity of $\mathbb{C}$. \\ $G(K / F) \quad$ the Galois group of a Galois extension $K / F$. \\ $S(F) \quad$ the set of all finite or infinite primes of $F$. \\ $S(K / F) \quad$ the set of those primes of $F$ which have at least a prime divisor \\ of degree 1 in a finite extension $K / k$. \\ $\hat{S}(K / F) \quad$ the set of those primes of $K$ which are of degree 1 in a finite \\ extension $K / F$. \\ $(A ; B) \quad$ the index of the subgroup $B$ in the group $A$ or the degree of \\ an extension field $A / B$. \\ $\bar{A} \quad$ the closure of $A$ in $J$ if $A$ is a subset of $J$, \\ $A \cdot B$ the set of all products of elements from $A$ and $B$, if $A$ and $B$ \\ are subsets of a group.
}

\title{
§ 1. Projection and restriction of idèle groups
}

Let $S=S(F)$ be the set of all primes of $F, s$ be a subset of $S$ containing infinitely many elements, and $s^{\prime}$ be its complement in $S: s^{\prime}=S-s$. We denote by $J_{s}$ the restricted direct product (as topological group) of $\mathfrak{p}$-adic completions $F_{\mathfrak{p}}$ over $\mathfrak{p}$-adic unit groups $U_{\mathfrak{p}}$ of $F$ where $\mathfrak{p}$ runs over $s$, that is, the group of all elements of the direct product $\prod_{\mathfrak{p} \in s} F_{\mathfrak{p}}$ whose $p$-components belong to $U_{\mathfrak{p}}$ for almost all $\mathfrak{p}$ of $s$. Denote also by $U_{s}$ the unit group $\prod_{p \in s} U_{\mathfrak{p}}$ of $J_{s}$. If $s=S$, then $J_{s}=J_{s}$ is the ordinary idèle group of $F$. We shall call an element of $J_{s}$ resp. of $U_{s}$ a s-restricted idèle resp. s-restricted unit idele.

We have algebraically and topologically

$$
J \cong J_{s} \times J_{s^{\prime}} \quad(\text { direct }) .
$$

Throughout this paper we shall fix this isomorphism, and the notation $\times$ is used for this direct product. Denote by $\pi_{s}$ the projection of $J$ to $J_{s}$, which is a multiplicative and open continuous map and is called s-projection. For any $\mathfrak{D} \in J$ we set always $\mathfrak{D}_{s}=\pi_{s}(\mathfrak{b})$. Put $F_{s}=\pi_{s}\left(F^{\times}\right), C_{s}=J_{s} / F_{s}$. By $\mathfrak{c}_{\mathrm{p}}, \mathfrak{c}_{\mathfrak{p}, s}$ and $\iota_{s}$ we mean the natural injections of $F_{\mathrm{p}}$ to $J, F_{\mathfrak{p}}$ to $J, F_{\mathfrak{p}}$ to $J_{s}$, and $J_{s}$ to $J$, respectively, 
These notations are omitted frequently when it can be done without danger of misunderstanding.

We define the s-restrtction $\rho_{s}$ by

$$
\rho_{s}(A)=\pi_{s}\left(A \cap \epsilon_{s} J_{s}\right)
$$

for any subset of $A$ of $J$. If an idèle $a \in J$ is not contained in $\iota_{s} J_{s}$, we write $\rho_{s}(\mathfrak{a})=\phi$. Then $\rho_{s}(\mathfrak{a} \mathfrak{b})=\rho_{s}(\mathfrak{a}) \rho_{s}(\mathfrak{b})$ for $\mathfrak{a}, \mathfrak{b} \in J$ if $\rho_{s}(\mathfrak{a}) \neq \phi$ and $\rho_{s}(\mathfrak{b}) \neq \phi$.

Lemma 1. Let $A$ be any subgroup of $J$.

Then

$$
\left({ }_{s s} J_{s}\right) A=J_{s} \times \pi_{s^{\prime}}(A) \quad(\text { direct }) .
$$

Proof. Let $\mathfrak{b} \mathfrak{b} \in A\left(\mathfrak{c}_{s} J_{s}\right), \mathfrak{a} \in A, \mathfrak{b} \in \imath_{s} J_{s}$. Since $\mathfrak{a}=\mathfrak{a}_{s} \times \mathfrak{a}_{s^{\prime}}$ and $\mathfrak{b}=\mathfrak{b}_{s} \times 1$, we have $\mathfrak{a} \mathfrak{b}=(\mathfrak{a} \mathfrak{b})_{s} \times \mathfrak{a}_{s^{\prime}} \in J_{s} \times \pi_{s^{\prime}}(A)$. Conversely let $\mathfrak{b}_{s} \in J_{s}$ and $\mathfrak{a} \in A$. Then $\mathfrak{b}_{s} \times \mathfrak{a}_{s^{\prime}}$ $=\left(\mathfrak{a}_{s} \times \mathfrak{a}_{s^{\prime}}\right)\left(\mathfrak{b}_{s} \mathfrak{a}_{s}^{-1} \times 1\right) A\left(\iota_{s} J_{s}\right)$. This lemma implies algebraically

$$
\frac{J_{s}}{\rho_{s}(A)}=\frac{\iota_{s} J_{s}}{A \cap \iota_{s} J_{s}} \cong \frac{\left(\iota_{s} J_{s}\right) A}{A}=\frac{J_{s} \times \pi_{s^{\prime}}(A)}{A}
$$

If $(J ; A)$ is finite, then $\left(J_{s} ; \rho_{s}(A)\right)=\left(J_{s} \times \pi_{s^{\prime}}(A) ; A\right)$

$$
=\frac{(J ; A)}{\left(J ; J_{s} \times \pi_{s}(A)\right)}=\frac{(J ; A)}{\left(J_{s} ; \pi_{s^{\prime}}(A)\right)} \text {. Hence we have }
$$

Lemma 2. Let $A$ be a subgroup of $J$ of finite index. Then

$$
\begin{aligned}
(J ; A) & =\left(J_{s} ; \pi_{s}(A)\right)\left(J_{s^{\prime}} ; \rho_{s^{\prime}}(A)\right) \\
& =\left(J_{s} ; \rho_{s}(A)\right)\left(J_{s^{\prime}} ; \pi_{s^{\prime}}(A)\right) .
\end{aligned}
$$

Lemma 3. Let $A$ be any subgroup of $J$ and $B$ be any subgroup of $J_{s}$. Then

$$
\rho_{s}\left(A \cdot\left(\iota_{s} B\right)\right)=\rho_{s}(A) \cdot B .
$$

Proof. Let $\mathfrak{a} \in A$ and $\mathfrak{b} \in \iota_{s} B$, then $\mathfrak{a} \mathfrak{b}=\left(\mathfrak{a}_{s} \times \mathfrak{a}_{s^{\prime}}\right)\left(\mathfrak{b}_{s} \times 1\right)=\mathfrak{a}_{s} \mathfrak{b}_{s} \times \mathfrak{a}_{s^{\prime}}$. Hence $\rho_{s}(\mathfrak{a b})=\mathfrak{a}_{s} \mathfrak{b}_{s}$ or $=\phi$ according to $a_{s},=1$ or $\neq 1$, that is, $\rho_{s}(\mathfrak{a})=\mathfrak{a}_{s}$ or $=\phi$. Hence $\rho_{s}\left(A\left(\ell_{s} B\right)\right) \subset \rho_{s}(A) B$. Conversely let $\mathfrak{a} \in A, \mathfrak{b} \in B$ and $\rho_{s}(\mathfrak{a}) \neq \phi$. Then $\rho_{s}(\mathfrak{a}) \mathfrak{b}=$ $\rho_{s}(\mathfrak{a}) \rho_{s}\left(\iota_{s} \mathfrak{b}\right)=\rho_{s}\left(\mathfrak{a}\left(\iota_{s} \mathfrak{b}\right)\right) \in \rho_{s}\left(A\left(\iota_{s} B\right)\right)$.

Lemma 4. Let $A$ be a subgroup of $J, B^{\prime}$ be a subgroup of $S_{s^{\prime}}$, and suppose $A \supset \iota_{\iota^{\prime}} B^{\prime}$. Then $\rho_{s}(A) \supset \pi_{s}\left(A \cap\left(J_{s} \times B^{\prime}\right)\right)$.

Proof. Let $a \times \mathfrak{b} \in A \cap\left(J_{s} \times B^{\prime}\right)$, where $\mathfrak{a} \in J_{s}$ and $\mathfrak{b} \in B^{\prime}$. Then $\pi_{s}(\mathfrak{a} \times \mathfrak{b})=\mathfrak{a}$ $=\pi_{s}\left((\mathfrak{a} \times \mathfrak{b})\left(1 \times \mathfrak{a}^{-1}\right)\right)$. Since $a \times \mathfrak{b} \in A$ and $1 \times \mathfrak{b}^{-1} \in A$, we have $a \in \pi_{s}\left(A \cap \iota_{s} J_{s}\right)$ $=\rho_{s}(A)$. 


\section{§ 2. Restricted idèles and the class field theory}

Let $k$ be any algebraic number field of finite degree, $K / k$ be a normal extension of finite degree, and $M$ be an abelian extension over $K$ which is normal over $k$. Let us characterize such $M$ by the restricted idèles attached to $K / k$.

The following propositions are well known.

PRoposition 1. (Bauer ${ }^{1)}$ ) Let $K$ be any normal extension of $k$ of finite degree and $\Omega$ be any extension of $k$ of finite degree. Then $S(K / k)$ contains $S(\Omega / k)$ if and only if $K$ is contained in $\Omega$.

We have immediately

Proposition 1'. Let $K_{1}$ and $K_{2}$ be normal extensions of finite degree. Then $S\left(K_{1} / k\right)=S\left(K_{2} / k\right)$ if and only if $K_{1}=K_{2}$.

Proposition 2. (Whaples ${ }^{2)}$ ). Let $A$ be any abelian extension of $F$ of finite degree, and $H$ be the subgroup of the idele group $J=J_{F}$ corresponding to $A$ by the class field theory. Let $\mathfrak{p}$ be a prime ideal of $F$. Then $\mathfrak{p}$ is not ramified in $A$ if and only if ${ }_{\mathfrak{p}} U_{\mathfrak{p}} \subset H$, and $\mathfrak{p}$ decomposes completely in $A$ if and only if ${ }_{\mathfrak{p}} F_{\mathfrak{p}} \subset H$.

Now we prove the following

Proposition 3. Let $K$ be a normal extension of $k$ of finite degree and put $\hat{s}=\hat{S}(K / k)$. Let $M_{1}$ and $M_{2}$ be abelian extensions of $K$ which are normal and of finite degree over $k$. Let further $H_{1}$ and $H_{2}$ be subgroups of the idele group of $K$ corresponding to $M_{1}$ and $M_{2}$, respectively, by the class field theory. Then we have $\rho_{\hat{s}}\left(H_{1}\right)=\rho \hat{s}\left(H_{2}\right)$ if and only if $H_{1}=H_{2}$, namely if and only if $M_{1}=M_{2}$.

Proof. It is obvious that $p \in S\left(M_{1} / k\right)$ if and only if $p \in S(K / k)$ and $p \in$ $S\left(M_{1} / K\right)$ for a prime divisor $\mathfrak{P}$ of $\mathfrak{p}$ in $K$. On the other hand $\mathfrak{P} \in S\left(M_{1} / K\right)$ if and only if $\iota_{\Re} K_{\Re} \subset H_{1}$ by prop. 2. Assume $\mathfrak{p} \in S(K / k)$, then $\iota_{\Re} K_{\Re} \subset H_{1}$ if and only if $\mathfrak{r}_{\mathfrak{R}} K_{\mathfrak{\beta}} \subset \rho_{\hat{s}}\left(H_{1}\right)$ for any prime divisor $\mathfrak{P}$ of $\mathfrak{p}$ in $K$. Hence $S\left(M_{1} / k\right)$ consists of all $\mathfrak{p} \in S(K / k)$ such that ${ }_{\mathfrak{\beta}} K_{\mathfrak{\beta}} \subset \rho \hat{s}\left(H_{1}\right)$ for any $\mathfrak{P}$ diving $\mathfrak{p}$. Therefore $\rho \hat{s}\left(H_{1}\right)=\rho_{\hat{s}}\left(H_{2}\right)$ implies $S\left(M_{1} / k\right)=S\left(M_{2} / k\right)$, and this implies $M_{1}=M_{2}$, hence $H_{1}=H_{2}$, by prop. $1^{\prime}$. Conversely if $H_{1}=H_{2}$, obviousely $\rho_{\hat{s}}\left(H_{1}\right)=\rho_{\hat{s}}\left(H_{2}\right)$. Thus the proposition is proved.

1) See M. Bauer [3], H. Hasse [10], $\$ 25$, and also M. Deuring [4].

2) See G, Whaples [13], also E. Artin and J. Tate [2], Ch. 8. 
Now let $F$ be any algebraic number field of finite degree, $J$ be the ordinary idèle group of $F$, and $\widehat{S}=J / F^{\times}$be its idèle class group. Denote by $\mathfrak{D}$ the connected component of the unity of $\mathfrak{S}$, and by $\mathscr{D}$ the complete inverse image of $\mathfrak{D}$ by the natural homomorphism of $J$ to $\sqrt{ }$. Let $s$ be an infinite set of primes of $F$ and $J_{s}$ be the $s$-restricted idèle group of $F$. We shall call a subgroup $H_{s}$ of $J_{s} s$-admissible if $H_{s}=\rho_{s}\left(\overline{H_{s} D}\right)$. If $H_{s}$ is $s$-admissible, then it is closed in $J_{s}$. In the case where $s$ is equal to the set $S=S(F)$ of all primes of $F$, we call simply admissible instead of $S$-admissible. There is a one to one correspondence between the set of admissible subgroups of $J$ and the set of class fields over $F$.

Let again $K / k$ be a normal extension of finite degree with the Galois group $G(K / k)$, and put $\hat{s}=\hat{S}(K / k)$. For each $\hat{s}$-restricted idèle $a \in J \hat{s}$ and each $\sigma \in$ $G(K / k)$, we define $\mathfrak{a}^{s}$ as the $\hat{s}$-restricted idèle whose $\mathfrak{p}^{\sigma}$-component is determined by $\left(\mathfrak{a}^{\mathfrak{p}}\right)_{\mathfrak{p} \sigma}=\left(\mathfrak{a}_{\mathfrak{p}}\right)^{\mathfrak{s}}$, where $\mathfrak{p}$ is a prime of $K$.

THEOREM 1. Let $K$ be a normal extension of $k$ of finite degree and put $G=G(K / k)$, and $\hat{s}=\hat{S}(K / k)$. Then the $\hat{s}$-restriction $\rho_{\hat{s}}$ gives a one to one correspondence between the set of all G-invariant admissible subgroups $H$ of $J$ of finite index and the set of all G-invariant $\hat{s}$-admissible subgroups $H_{\hat{s}}$ of $J_{\hat{s}}$ of finite index. Futhermore if $\rho_{\hat{s}}(H)=H \hat{s}$, then we have $G$-isomorphism $J / H \cong J_{\hat{s}} / H_{\hat{s}}$.

Proof. Let $H$ be a $G$-invariant admissible subgroup of $J$ of finite index. Then $\rho_{\hat{s}}(H)$ is obviousely $G$-invariant. Since $H$ contains both $\rho_{\hat{s}}(H)$ and $D$, and

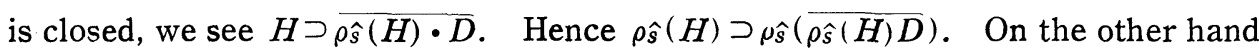
obviousely $\rho_{\hat{s}}(H) \subset \rho_{\hat{s}}\left(\overline{\rho_{\hat{s}}(H) D}\right)$. Thus $\rho \hat{s}(H)$ is $\hat{s}$-admissible. Moreover we have $(J ; H)=\left(J_{\hat{s}} ; \rho \hat{s}(H)\right)\left(J_{\hat{s}}, ; \pi \hat{s},(H)\right)$ by lemma 2 , and this implies that $\rho \hat{s}(H)$ is of finite index as a subgroup of $J \hat{s}$. Conversely let $H \hat{s}$ be a $G$-invariant $\hat{s}$-admissible subgroup of $J \hat{s}$ of finite index. Put $H=\overline{H_{\hat{s}} \cdot D}$. Then $H$ is $G$-invariant and admissible. Since $H_{\hat{s}}$ is $\hat{s}$-admissible, $\rho \hat{s}(H)=H_{\hat{s}}$. Thus if we prove the finiteness of $(J ; H)$, then the first part of the theorem follows from prop. 3. So we shall prove the finiteness. Put $H_{1}=\overline{J_{\hat{s}}} \bar{D}$. Then $H_{1}$ is $G$-invariant and admissible subgroup of $J$. Moreover if $\mathfrak{P}$ is contained in $\hat{s}$, then $\mathfrak{\wp}_{\mathfrak{p}} K_{\mathfrak{\beta}}$ is contained in $H_{1}$. Let $A_{1}$ be the abelian extension over $K$ corresponding to $H_{1}$ by the class field theory. Then $A_{1}$ is normal over $k$ and we have $S\left(A_{1} / k\right) \supset S(K / k)$ by prop. 2 . Hence $A_{1} \subset K$ by prop. 1. We have necessarily $A_{1}=K$, hence $H_{1}$ 
$=\overline{J_{\hat{s}} D}=J$. Thus we see algebraically $J / H=\overline{J_{\hat{s}} D} / H \subset J_{\hat{s}} H / H \cong J_{\hat{s}} / J_{\hat{s}} H=J_{\hat{s}} / J_{\hat{s}}(H)$ $=J \hat{s} / H_{\hat{s}}$. Since $\left(J_{\hat{s}} ; H_{\hat{s}}\right)$ is finite, $(J ; H)$ is finite. Thus the finiteness and so the one to one correspondence in the theorem is proved.

Now let again $H$ be a $G$-invariant admissible subgroup of $J$ of finite index. Since the restriction $\pi \hat{s}^{\prime}$ is an open map of $J$ to $J \hat{s}^{\prime}, \pi \hat{s}^{\prime}(H)$ is closed in $J \hat{s}^{\prime}$. Moreover $\pi \hat{s},(H)$ is obviousely $G$-invariant and contains $\pi \hat{s}^{\prime}(D)$. Put $J_{\hat{s}} \times \pi \hat{s}^{\prime}(H)$ $=H_{1}$. Then $\left(J ; H_{1}\right)$ is finite and $H_{1}$ is a $G$-invariant admissible subgroup of $J$. Let $A_{1}$ be the class field over $K$ corresponding to $H_{1}$. Then $A_{1}$ is a normal extension over $k$ and $S\left(A_{1} / K\right) \supset \hat{s}=\hat{S}(K / k)$ by prop. 2, hence $S\left(A_{1} / k\right) \supset S(K / k)$. This implies $A_{1} \subset K$ by prop. 1 . Then we have necessarily $A_{1}=K$, which implies $J_{\hat{s}^{\prime}}=\pi_{\hat{s}^{\prime}}\left(H_{1}\right)$. Since $\pi \hat{s}^{\prime}\left(H_{1}\right)=\pi \hat{s}^{\prime}(H)$, we have $J_{\hat{s}^{\prime}}=\pi \hat{s}^{\prime}(H)$. Hence $J / H \cong J \hat{s} / H \hat{s}$ by (3).

Thus the theorem is completely proved.

The following theorem is an immediate consequence of the class field theory and theorem 1 .

TheOREM 2. Let $K$ be a normal extension of $k$ of finite degree and put $G=G(K / k), \hat{s}=\hat{S}(K / k)$. Then there is a one to one correspondence between the set of all $G$-invariant $\hat{s}-a d m i s s i b l e ~ s u b g r o u p s ~ H \hat{s}$ of $J_{\hat{s}}$ of finite index and the set of all abelian extensions $M$ of $K$ of finite degree which is normal over $k$. If $M$ corresponds to $H_{\hat{s}}$, then $G(M / K) \cong J \hat{s} / H_{\hat{s}}$. Furthermore a prime $\mathfrak{B}$ of $\hat{s}$ decomposes completely in $M$ if and only if $\iota_{\mathfrak{p}} K_{\mathfrak{p}} \subset H_{\hat{s}}$.

\section{$\S 3$. Conditions of the s-admissibility}

Let $F$ be any algebraic number field, $J$ be the idèle group of $F,\left[5=J / F^{\times}\right.$ be its idèle class group, and $D$ be the connected component of the unity in 5 . Let further $s$ be a set of infinitely many primes of $F$ and $J_{s}$ be the restricted idèle group of $F$. We have called, in the previous section, a subgroup of $H_{s}$ of $J_{s} s$-admissible if $H_{s}=\rho_{s}\left(\bar{H}_{s} \bar{D}\right)$ where $D$ is the complete inverse image of $\mathbb{T}$ by the natural homomorphism of $J$ to $\$$. Now we prove

THEOREM 3. Let $H_{s}$ be a closed subgroup of $J_{s}$ of finite index. Then $H_{s}$ is s-admissible if and only if there exists a neighborhood $U^{\prime}$ of the unity in $J_{s}$, such that $H_{s}$ contains $\pi_{s}\left(D \cap\left(J_{s} \times U^{\prime}\right)\right)$.

Proof. Suppose that $H_{s}$ is $s$-admissible, and put $H=\overline{H_{s} D}$. Then $H$ is ad- 
missible in $J$ and $\rho_{s}(H)=H_{s}$. Let $U$ be a neighborhood of the unity in $J$ whith $H \supset U$. Such a neighborhood always exists because of the finiteness of $(J ; H)$. Set $U=U_{s_{.}} \times U_{s^{\prime}}$ where $U_{s} \subset J_{s}$ and $U_{s^{\prime}} \subset J_{s^{\prime}}$. Then $U_{s^{\prime}}$ is a neighborhood of the unity in $J_{s^{\prime}}$ and we see $H_{s}=\rho_{s}(H) \supset \pi_{s}\left(H \cap\left(J_{s} \times U_{s^{\prime}}\right) \supset \pi_{s}\left(D \cap\left(J_{s} \times U_{s^{\prime}}\right)\right)\right.$ by lemma 4.

Suppose conversely that there exists a neighborhood $U^{\prime}$ of the unity in $J_{S^{\prime}}$ such that $H_{s} \supset \pi_{s}\left(D \cap\left(J_{s} \times U^{\prime}\right)\right)$. Let $\mathfrak{x}$ be any element of $\rho_{s}\left(\overline{H_{s}} \bar{D}\right)$, then $\mathfrak{x} \times 1 \in$ $\overline{H_{s} D}$. Now let $U(\mathfrak{x})$ be any neighborhood of $\mathfrak{x}$ in $J_{s}$ and put $U=U(\mathfrak{x}) \times U^{\prime}$. Then since $U$ is a neighborhood of $\mathfrak{x} \times 1$ in $J, U \cap\left(H_{S} D\right)$ is not empty. Let $(\mathfrak{h} \times 1)$ $(\mathfrak{a} \times \mathfrak{b})$ be in this intersection, where $\mathfrak{h} \in H_{s}, \mathfrak{a} \times \mathfrak{b} \in D, \mathfrak{a} \in J_{s}, \mathfrak{b} \in J_{\mathfrak{s}^{\prime}}$. Then $(\mathfrak{b} \times 1)(\mathfrak{a} \times \mathfrak{b})=\mathfrak{h} \mathfrak{a} \times \mathfrak{b} \in U, \mathfrak{b} \in U^{\prime}$ and $\mathfrak{h} \mathfrak{a} \in U(\mathfrak{x})$. Hence $\mathfrak{a} \in \pi_{s}\left(D \cap\left(J_{s} \times U^{\prime}\right)\right) \subset H_{s}$ and $\mathfrak{h a} \in H_{s}$. Therefore $U(\mathfrak{x}) \cap H_{s}$ is not empty, which implies $\mathfrak{x} \in \bar{H}_{s}=H_{s}$, since $H_{s}$ is closed in $J_{s}$. Thus we have $\rho_{s}\left(\overline{H_{s} D}\right) \subset H_{s}$, hence $\rho_{s}\left(\overline{H_{s} D}\right)=H_{s}$. This proves that $H_{s}$ is $s$-admissible, and the theorem is proved.

Now the structure of $D$ is known by Artin $[1]^{3)}$. Let $\mathbf{Z}$ be the additive group of rational integers and $\overline{\mathbf{Z}}$ be the completion of $\mathbf{Z}$ under the topology where the ideals of $\mathbf{Z}$ form a fundamental system of neighborhoods of 0 . For each unit $\varepsilon$ of $F$, we denote by $\bar{\varepsilon}$ the idèle which has components $\varepsilon$ at all finite primes and components 1 at all infinite primes. Then for $x \in \overline{\mathbf{Z}}, \bar{\varepsilon}^{x}$ can be defined as the extension of the exponentiation $\varepsilon^{m}$ by an ordinary integer $m$. Let $r_{1}, r_{2}$ be as usual the numbers of real primes and complex primes of $F$ respectively and put $r=r_{1}+r_{2}-1$. Then we have by Artin [1] immediately the following

PROPOSITION 4. Let $\varepsilon_{1}, \varepsilon_{2}, \ldots, \varepsilon_{r}$ be any system of independent totally positive units of $F$. Let further $E$ be the group of all ideles $\bar{\varepsilon}^{x_{1}} \cdots \bar{\varepsilon}_{r}^{\lambda_{r}}$ where $x_{1} \ldots, x_{r} \in \overline{\mathbf{Z}}$, and $J_{\infty}$ be the group of those ideles which have components 1 at all finite primes. Then we can choose a system of representatives of $\mathrm{D}$ mod. $F^{\times}$ in $E J_{\infty}$.

Notations being as above, let $x \in \overline{\mathbf{Z}}$ and $\varepsilon$ be one of the $\varepsilon_{i}$. Let further $U^{\prime}$ be a neighborhood of the unity in $J_{s^{\prime}}$ containing $\pi_{s^{\prime}}\left(J_{\infty}\right)$. Then there exists an integer $m \in \mathbf{Z}$ such that $\bar{\varepsilon}^{x} / \varepsilon^{m} \in J_{s} \times U^{\prime}$. Indeed let $\mathfrak{p}$ be a finite prime. If $x^{\prime} \equiv 0$ mod $p^{\kappa}$ for a sufficiently large $\kappa$, then the $\mathfrak{p}$-component of the idèle $\bar{\varepsilon}^{x^{\prime}}$ is in the $\mathfrak{p}$-component of $J_{s} \times U^{\prime}$. Since the $\mathfrak{p}$-component of $J_{s} \times U^{\prime}$ containes $U_{\mathfrak{p}}$ for almost

3) See also E. Artin and J. Tate [2], Ch. 9. 
all $\mathfrak{p}$, we can set $\kappa=0$ for such almost all $\mathfrak{p}$. This implies immediately the existence of the above integer $m$, being $x \equiv m \bmod$. $\mathfrak{p}^{\kappa}$ for all finite $\mathfrak{p}$. Hence by prop. 4 we have

$$
\left(D \cap\left(J_{s} \times U^{\prime}\right)\right) \cdot F=D .
$$

Lemma 5. Let $G$ be a topological group which is locally compact and satisfies the second axiom of countability. Let $N$ be a discrete normal subgroup and $A$ be a closed subgroup of $G$. Suppose further $A N$ is closed in $G$. Then we have $A N / N \cong A / A N$.

Proof. Let $f$ be the canonical map of $G$ to $G / N$. Then $f$ is an open map, and we have $f(A)=f(A N)=A N / N$. Since $N$ is discrete, $G / N$ is locally isomorphic to $G$ ، Hence $G / N$ is also locally compact and satisfies the second axiom of countability. Both $A$ and $A N$ are locally compact, because they are closed in $G$. Furthermore $A N / N$ is also locally compact owing to the continuity of $f$. It is known $n^{4}$ that if $g$ is an onto-homomorphic mapping between two locally compact groups satisfying the second axiom of countability, then $f$ is open. Therefore the restriction of $f$ to $A$, whose image is $A N / N$, is also open. Then since the kernel of $f$ is $A N$, we have $f(A)=A / A N$. Thus the lemma is proved.

PROPOSITION 5. Let $U^{\prime}$ be a neighborhood of the unity in $J_{S^{\prime}}$ containing $\pi_{s^{\prime}}\left(J_{\infty}\right)$, and $H_{s}$ be a closed subgroup of $J_{s}$ of finite index. If $H_{s} \supset \pi_{s}\left(F^{\times} \cap\left(J_{s} \times U^{\prime}\right)\right)$, then $\left.H_{s} \supset \pi_{s}\left(D \cap \times U^{\prime}\right)\right)$.

Proof. We have the following commutative diagram

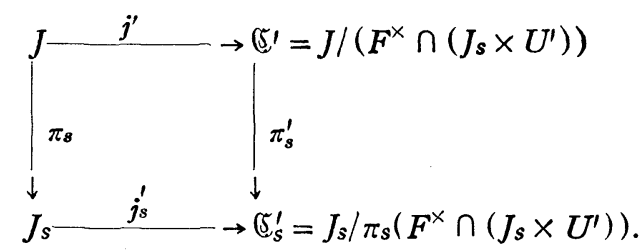

where $j^{\prime}$ and $j_{s}^{\prime}$ are the canonical homomorphisms respectively. First we shall prove that $j_{s}^{\prime} \pi_{s}\left(D \cap\left(J_{s} \times U^{\prime}\right)\right)$ is connected in $\mathbb{E}_{s}^{\prime}$. Since $J, F^{\times}$and $\left(D \cap\left(J_{s} \times U^{\prime}\right)\right)$ satisfy the conditions of $G, N$ and $A$ in lemma 5 respectively, we have alge-

4) See L. S. Pontrjagin [12], Ch. $3, \S 19$, theorem 13. In this book, the topology is $T_{1}$ and every subgroup is closed. But the proof of the theorem 13 in this book hold in general case. 
braically and topologioally $D / F^{\times}\left(D \cap\left(J_{s} \times U^{\prime}\right)\right) F^{\times} / F^{\times} \cong D \cap\left(J_{s} \times U^{\prime}\right)=j^{\prime}(D \cap$ $\left.\left(J_{s} \times U^{\prime}\right)\right)$. Hence $j^{\prime}\left(D \cap\left(J_{s} \times U^{\prime}\right)\right)$ is connected in $\left(\xi_{s}^{\prime}\right.$, because $D / F^{\times}=\mathscr{D}$ is the connected component of the unity in $\left(5\right.$. Since $j^{\prime}, j_{s}^{\prime}, \pi_{s}$ are all continuous maps, $\pi_{s}^{\prime}$ is also continuous and we have $\pi_{s}^{\prime} j^{\prime}\left(D \cap\left(J_{s} \times U^{\prime}\right)\right)=j_{s}^{\prime} \pi_{s}\left(D \cap\left(J_{s} \times U^{\prime}\right)\right)$. Hence $j_{s}^{\prime} \pi_{s}\left(D \cap\left(J_{s} \times U^{\prime}\right)\right)$ is connected in $\mho_{s}^{\prime}$. Now since $H_{s}$ is closed in $J_{s}$ and contains $\pi_{s}\left(F^{\times} \cap\left(J_{s} \times U^{\prime}\right)\right), j_{s}^{\prime}\left(H_{s}\right)$ is also closed in $\xi_{s}^{\prime}$. On the other hand, $j_{s}^{\prime}\left(H_{s}\right)$ is of finite index in $\mathfrak{G}_{s}^{\prime}$, since $H_{s}$ is so in $J_{s}$. Hence $j_{s}^{\prime}\left(H_{s}\right)$ is open in $\Xi_{s}^{\prime}$. Therefore $j_{s}^{\prime}\left(H_{s}\right)$ must contain $j_{s}^{\prime} \pi_{s}\left(D \cap\left(J_{s} \times U^{\prime}\right)\right)$ which is connected in $\mho_{s}^{\prime}$ as proved above. This implies $H_{s} \supset \pi_{s}\left(D \cap\left(J_{s} \times U^{\prime}\right)\right)$, since $H_{s} \supset \pi_{s}\left(F^{\times} \cap\left(J_{s} \times U^{\prime}\right)\right)$ by the assumption. Thus the proposition is proved.

Let again $K$ be a normal extension of $k$ of finite degree, and let $J=J_{K}$. Put $s=\hat{S}(K / k), G=G(K / k)$. Then we have

Theorem 4. Let $H \hat{s}$ be a G-invariant closed subgroup of $J_{\hat{s}}$ of finite index. Then, in order that $H_{\hat{s}}$ is $\hat{s}-a d m i s s i b l e$, it is necessary and sufflcient that there exists a noighborhood $U^{\prime}$ of the unity in $J_{\hat{s}}$ such that $U^{\prime} \supset \pi \hat{s},\left(J_{\infty}\right)$ and $H_{\hat{s}} \supset \pi \hat{s}$ $\left(K^{\times} \cap\left(J_{\hat{s}} \times U^{\prime}\right)\right)$.

Proof. Suppose that $H \hat{s}$ be $s$-admissible and put $H=\overline{H_{s} D}$. Then $\rho_{\hat{s}}(H)$ $=H \hat{s}$. If $K=k$, then $\hat{s}^{\prime}$ is empty and the theorem is true by the class field theory. Thus we assume $K \neq k$. As the proof of theorem 3, let $U$ be a neighborhood of the unity in $J$ such that $H \supset U$ and set $U=U_{\hat{s}} \times U_{\hat{s}}$, where $U_{\hat{s}} \subset J_{\hat{s}}$, $U_{\hat{s}}, \subset J_{\hat{s}}$, . Then we can assume that $U_{\hat{s}^{\prime}}$ contains $\pi \hat{s}^{\prime}\left(J_{\infty}\right)$, because every inninite prime of $\hat{s}^{\prime}$ is ramified in $K / k$, and consequently complex. Hence it is not ramified in any extension over $K$. Therefore, if $\mathfrak{p}$ is an infinite prime of $\hat{s}^{\prime}$, then $U_{\mathfrak{p}}$ is contained in $H$. This implies $U_{\infty}^{\prime} \subset H$. Now we see, as the proof of the theorem 3, that $H \hat{s}=\rho \hat{s}(H) \supset \pi \hat{s}\left(H \cap\left(J_{s} \times U_{\hat{s}},\right)\right) \supset \pi \hat{s}\left(K \cap\left(J_{\hat{s}} \times U_{\hat{s}},\right)\right.$, which proves the necessity of the theorem. The sufficiency follows immediately from theorem 3 and prop. 5.

\section{$\S 4 . \quad k$-meta-abelian extensions}

Let $K / k$ be, as in the previous sections, an extension of degree $n$ of an algebraic number field $k$, and put $s=S(K / k), \hat{s}=\hat{S}(K / k), G=G(K / k)$. For every prime $\mathfrak{p}$ of $k$ we fix a prime $\mathfrak{P}$ of $K$ which divides $\mathfrak{p}$, and set $(\mathfrak{p}, \sigma)=\mathfrak{P}^{\sigma}$ for $\sigma \in G$. Then $\hat{s}$ consists of all $(\mathfrak{p}, \sigma)$ such that $\mathfrak{p} \in s$ and $\sigma \in G$. Denote by $\hat{s}(\sigma)$ the set of all $(\mathfrak{p}, \sigma)$ where $\mathfrak{p} \in s$. Then $\hat{s}$ is the union of those $\hat{s}(\sigma)$ where $\sigma \in G$, and 
we have naturally

$$
J_{\hat{s}} \cong \prod_{\sigma \in G} J_{\hat{s}}(\sigma) \quad \text { (direct) } .
$$

Hereafter we shall fix this isomorphism.

If $\mathfrak{p} \in \hat{s}$, any element $\alpha$ of $K_{\mathfrak{\beta}} \sigma$ is expressed by $\alpha=\sum a_{i} \pi^{i}$ where $a_{i} \in k$ and $\pi$ is any fixed prime element of $k_{\mathfrak{p}}$. Thus the element of $K_{\mathfrak{p} \sigma}$ is naturally embedded in $k_{\mathfrak{p}}$. We denote this embedding by $g_{\mathfrak{p} \sigma}$ or $g_{(\mathfrak{p}, \sigma)}$, and we have $g_{\mathfrak{P}} \sigma\left(\alpha^{\sigma}\right)=g_{\mathfrak{\Re}}(\alpha)$ for $\alpha \in K_{\mathfrak{\beta}}, \sigma \in G$. Denote by $g_{\sigma}$ the mapping of $J \hat{s}_{(\sigma)}$ to $J_{s}$, which maps every $(\mathfrak{p}, \sigma)$-component by $g_{\mathfrak{p}} \sigma=g_{(\mathfrak{p}, \sigma)}$. Set $g=\prod_{\sigma \in G} g_{\sigma}$. Then $g_{\sigma}$ and $g$ imply algebraically and topologically

$$
J_{\hat{s}(\sigma)} \cong J_{s}
$$

and

$$
J_{\hat{s}} \cong J_{s}^{n},
$$

where $J_{s}^{n}$ is the direct product of $n$-fold of $J_{s}$.

Let $\sigma_{1}, \ldots, \sigma_{n}$ be all elements of $G$ and $P_{o}$ be the permutation $\left(\sigma_{1}, \ldots\right.$, $\left.\sigma_{n}\right) \rightarrow\left(\sigma_{1} \sigma, \ldots, \sigma_{n} \sigma\right)$ for every element $\sigma \in G$. For $\left(a_{1}, \ldots, a_{n}\right) \in J_{s}^{n}$ set

$$
\left(a_{1}, \ldots, a_{n}\right)^{\sigma}=P_{\sigma}\left(a_{1}, \ldots, a_{n}\right) .
$$

Then $J_{s}^{n}$ has $G$ as the operator domain, and the isomorphism ( $\sigma$ ) becomes a $G$-isomorphism.

Now denote by $\mathbf{A}$ the maximal abelian extension of $k$. Let $M$ be a metaabelian extension of $k$ of finite degree, that is, a normal extension of $k$ of finite degree with an abelian group as commutator group of the Galois group. Then $\mathbf{A} M / \mathbf{A}$ is a Kummer extension. Let $\mathbf{A} M=\mathbf{A}\left(\sqrt[m_{1}]{a_{1}}, \ldots, m_{t} \sqrt{a_{t}}\right)^{5}$. Then if every $a_{i}$ is an element of $k$, we called in [13] $M$ a $k$-meta-abelian field over $k$, and proved that $M$ contains necessarily the all $m_{i}$-th roots of unity $(i=1, \ldots, t)$. Therefore if $M$ is a $k$-meta-abelian field over $k$, there exists an abelian extension $K$ of $k$ which containes the $m_{i}$-th roots of unity and $K\left(\sqrt[m_{1}]{a_{1}}, \ldots, m_{t} \sqrt{a_{t}}\right) \supset M \supset K$ where $a_{1}, \ldots, a_{t} \in k$.

Put, as before, $s=S(K / k)$ and $\hat{s}=\hat{S}(K / k)$. For $a \in J_{K}$ and $a \in k$ let $(\mathfrak{a}, a)_{m}$ be the norm residue symbol for Kummer extension $K(\sqrt[m]{a}) / K^{6}$. Denote by $\left(a_{\mathfrak{p}}, a\right)_{\mathfrak{p}, m}$ the norm residue symbol for the local Kummer extension $K_{\mathfrak{P}}(\dot{m} \sqrt{a}) / K_{\mathfrak{P}}$.

5) We assume $n_{i} \sqrt{ } \overline{a_{i}} \notin A M$ for any integer $n_{i}>m_{i}$.

6) c. f. E. Artin and J. Tate [2], Ch. 12. 
Then we have

$$
(a, a)_{m}=\prod_{\mathfrak{F} \in \mathcal{S}(K)}\left(a_{\mathfrak{B}}, a\right)_{\mathfrak{F}, m} .
$$

Let $H_{m}(a)$ be the idele group of $\mathfrak{a} \in J_{K}$ such that $(\mathfrak{a}, a)_{m}=1$. Then $H_{m}(a)$ is the admissible subgroup of $J_{K}$ corresponding to $K(\sqrt[m]{a})$ by the classfield theory. We see immediately that $\rho \hat{s}\left(H_{m}(a)\right)$ consists of all $a \in J \hat{s}$ such that $(a, a)_{m}=1$, where $(\mathfrak{a}, \boldsymbol{a})_{m}=\prod_{\mathfrak{W} \in s}\left(\mathfrak{a}_{\mathfrak{p}}, \boldsymbol{a}\right)_{\mathfrak{w}, m}$. The isomorphism $g$ implies

$$
(a, a)_{m}=\prod_{\substack{\mathfrak{q} \in s \\ \sigma \in G}}\left(a_{(\mathfrak{p}, \sigma)}, a\right)_{m} \text { for } \mathfrak{a} \in J_{s}^{n}
$$

Now we shall call a subgroup $H_{1}$ of $J_{s}^{n} k$-admissible if there exist elements $a_{1}, \ldots, a_{t}$ of $k$ and natural numbers $m_{1}, \ldots, m_{t}$ such that $H_{1}$ contains the intersection of all $H_{m_{1}}\left(a_{1}\right), \ldots, H_{m_{t}}\left(a_{t}\right)$, provided that $s$ contains all primes of $k$ such that $N p-1$ is divisible by all $m_{1}, \ldots, m_{t}$. Then we have by theorem 1 the following theorem, which essentially includes the main part of Kuroda [11], Fröhlich [5] and Furuta [7], [8].

Theorem 5. Let $M$ be a k-meta-abelian extension over $k$ of finite degree. Then there exist an admissible closed subgroup $H$ of the (ordinary) idele group $J$ of $k$ of finite index, say $n$, and a $k$-admissible closed subgroup $H_{1}$ of the restricted idele group $J_{s}^{n}$ of $k$ of finite index which satisfies the following condition $(\#)$, where $s$ is the set of all primes $\mathfrak{p}$ of $k$ such that $\iota_{p} k_{\mathfrak{p}} \subset H$.

(\#) If $K$ is the class field over $k$ corresponding to $H$, we have $G(M / K)=$ $J_{s}^{n} / H_{1}$ and a prime $\mathfrak{p}$ of $k$ decomposes completely in $M / k$ if and only if $\mathfrak{p} \in s$ and $\iota_{p} k_{p} \subset H_{1}$.

Conversely let $H$ be an admissible closed subgroup of the (ordinary) idèle group $J$ of $k$ of finite index $n$ and $H_{1}$ be a k-admissible closed subgroup of the restricted idele group $J_{s}^{n}$ of $k$ of finite index, where $s$ is the set of all primes $p$ of $k$ such that $\varsigma_{\mathfrak{p}} k_{\mathfrak{p}} \subset H$. Then there exists a $k$-meta-abelian field $M$ over $k$ which satisfies the condition ( $\#)$.

\section{References}

[1] E. Artin, Representatives of the connected component of the idele class group, Proc. Int. Symp. Algebraic Number Theory, Tokyo-Nikko, 1955, 51-54.

[2 ] E. Artin and J. Tate, Class field theory, Princeton notes, 1951, distributed by Harvard University. 
[ 3 ] M. Bauer, Zur Theorie der algebraischen Zahlkörper, Math. Ann., 77 (1916).

[4] M. Deuring, Neuer Beweis des Bauerschen Satzes, J. reine und angew. Math., 173 (1935), 1-4.

[5] A. Fröhlich, The restricted biquadratic residue symbol, Proc. London Math. Soc., (3) 9 (1959), 189-207.

[6] A. Fröhlich, A prime decomposition symbol for certain non Abelian number fields, Acta Sci. Math., 21 (1960), 229-246.

[ 7 ] Y. Furuta, A reciprocity law of the power residue symbol, J. Math. Soc. Japan, 10 (1958), 46-54.

[ 8 ] Y. Furuta, On meta-abelian fields of a certain type, Nagoya Math. J., 14 (1959), 193199.

[ 9 ] Y. Furuta, A property of meta-abelian extensions, Nagoya Math. J., 19 (1961), 169187.

[10] H. Hasse, Bericht über neuere Untersuchungen und Probleme aus der Theorie der algebraischen Zahlkörper, II, Jber. Deutsch. Math. Verein., 35 (1926).

[11] S. Kuroda, Über die Zerlegung rationaler Primzahlen in gewissen nicht-abelschen galoischen Körpern, J. Math. Soc. Japan, 3 (1951), 148-156.

[12] L. S. Pontrjagin, Topological groups. Princeton (1939).

[13] G. Whaples, Non analytic class field theory and Grunwald's theorem, Duke Math. J., 9 (1942).

\section{Mathematical Institute \\ Kanazawa University}

